\title{
Relationships between the structural and functional organization of the turtle cell nucleolus
}

\author{
Odile Bartholoméa ${ }^{\text {, Claire Franck }}{ }^{\mathrm{a}}$, Patricia Piscicelli ${ }^{\mathrm{a}}$, Nathalie Lalun ${ }^{\mathrm{b}}$, Jean Defourny ${ }^{\mathrm{a}}$, \\ Justine Renauld $^{\mathrm{a}}$, Nicolas Thelen ${ }^{\mathrm{a}}$, Françoise Lamaye ${ }^{\mathrm{a}}$, Dominique Ploton ${ }^{\mathrm{b}}$, Marc Thiry ${ }^{\mathrm{a}}{ }_{*}$ \\ ${ }^{\text {a } U n i t ~ o f ~ C e l l ~ B i o l o g y, ~ G I G A-N e u r o s c i e n c e s, ~ U n i v e r s i t y ~ o f ~ L i e g e, ~ C H U ~ S a r t-T i l m a n, ~ B 36, ~} 4000$ Liege, Belgium \\ ${ }^{\mathrm{b}}$ UMRCNRS 6237, Université de Reims Champagne-Ardenne, 51 rue Cognacq-Jay, 51095 Reims Cedex, France
}

\section{A R T I C L E I N F O}

\section{Keywords:}

Nucleolus organization

Ribogenesis

Amniotes

Turtles

Reptiles

\begin{abstract}
A B S T R A C T
The nucleolus is a multifunctional structure of the eukaryotic cell nucleus. However, its primary role is ribosome formation. Although the factors and mechanisms involved in ribogenesis are well conserved in eukaryotes, two types of nucleoli have been observed under the electron microscope: a tricompartmentalized nucleolus in amniotes and a bicompartmentalized nucleolus in other species. A recent study has also revealed that turtles, although belonging to amniotes, displayed a nucleolus with bipartite organization, suggesting that this reptile group may have carried out a reversion phenomenon during evolution. In this study, we examine in great detail the functional organization of the turtle nucleolus. In liver and spleen cells cultured in vitro, we confirm that the turtle nucleolus is mainly formed by two components: a fibrillar zone surrounded by a granular zone. We further show that the fibrillar zone includes densely-contrasted strands, which are positive after silver-stained Nucleolar Organizer Region (Ag-NOR) staining and DNA labelling. We also reveal that the dense strands condensed into a very compact mass within the fibrillar zone after a treatment with actinomycin D or 5,6-dichlorobenzimidazole riboside. Finally, by using pulse-chase experiments with BrUTP, three-dimensional image reconstructions of confocal optical sections, and electron microscopy analysis of ultrathin sections, we show that the topological and spatial dynamics of rRNA within the nucleolus extend from upstream binding factor (UBF)-positive sites in the fibrillar zone to the granular zone, without ever releasing the positive sites for the UBF. Together, these results seem to clearly indicate that the compartmentalization of the turtle nucleolus into two main components reflects a less orderly organization of ribosome formation.
\end{abstract}

\section{Introduction}

The nucleolus is the most identifiable structure of the eukaryotic cell nucleus. Recent papers show that the nucleolus is implicated in many important events such as repair of DNA damage or cancer, highlighting its widespread role not only in ribosome formation but also in cell cycle control (Ogawa and Baserga, 2017; Penzo et al., 2019). The primary role of the nucleolus is the biogenesis of most cell ribonucleoprotein particles (Boisvert et al., 2007). As such, the size and organization of the nucleolus are directly related to the level of ribosome production (Hernandez-Verdun, 2006).

Ribosome biogenesis is a complex process that involves many successive steps (Lafontaine, 2004). Factors and mechanisms are well conserved in eukaryotes. It starts with the transcription of ribosomal genes by RNA polymerase I, which results in the production of a primary ribosomal RNA (rRNA). It then undergoes a maturation which involves modification of specific bases and a series of cleavages leading to mature 18S, $5.8 \mathrm{~S}$ and $28 \mathrm{~S}$ rRNA. During the maturation of rRNA, rRNA molecules associate with ribosomal and nonribosomal proteins as well as with 5S rRNA (transcribed by RNA polymerase III outside the nucleolus) to form two preribosomal particles which will be exported to the cytoplasm where they become the two subunits of ribosomes (Henras et al., 2015; Turowski and Tollervey, 2015).

Classically, the nucleolus is described as a structure made of three main compartments: the fibrillar centers (FC), the dense fibrillar component (DFC) and the granular component (GC) (Thiry and Goessens,

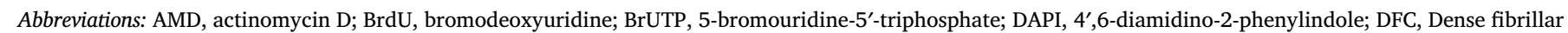

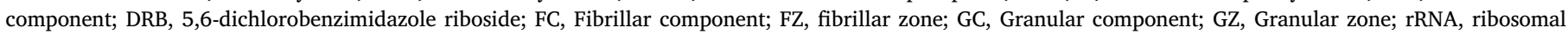
ribonucleic acid; UBF, upstream binding factor

* Corresponding author.

E-mail address: mthiry@uliege.be (M. Thiry).
} 
1996; Thiry and Lafontaine, 2005; Derenzini et al., 2006). FCs are round structures of variable size, presenting a very low electron opacity. They are partially surrounded by the DFC, a strongly contrasted material. These two compartments are included in the GC that mainly consists of granules $15-20 \mathrm{~nm}$ in diameter. It is generally admitted that FCs represent sites of transcription of non-nucleosomal rDNA loops. This is supported by the fact that major specific markers of r-chromatin, such as RNA polymerase I, UBF, DNA topoisomerase I (Thiry and Goessens, 1996, Seither et al., 2001; Thiry and Lafontaine, 2005; Derenzini et al., 2006), are localized inside FCs. The FC cortex is considered to contain transcriptionally active r-genes, from which the growing pre-rRNA enters into the DFC (Cheutin et al., 2002). However, some authors consider that active r-genes, together with linked Christmas tree segments, are exclusively confined to DFC (Koberna et al., 2002). Spatio-temporal studies have also revealed that DFC surrounding FC corresponded to the nucleolar zone where early processing of nascent pre-rRNA takes place (Thiry et al., 2000; Cheutin et al., 2002). This is also confirmed by the localization of early processing markers such as fibrillarin and nucleolin (Krüger et al., 2007). The late steps of rRNA maturation occur in the GC, as revealed by the presence of specific markers (B23, NOP52, ribosomal proteins).

This classical organisation can only been found in amniotes species while other eukaryotes possess nucleoli with only two distinct compartments (Thiry and Lafontaine, 2005). However, while crocodilians, lizards and snakes present a tricompartmentalized nucleolus, a detailed analysis of several reptiles revealed that turtles have also a bicompartmentalized nucleolus (Lamaye et al., 2011; Thiry et al., 2011; see also Fig. S1 for two new examples). Even if the place of turtle is not exactly defined in the phylogenetic tree (Müller 2003; Werneburg and Sánchez-Villagra 2009; Chiari et al., 2012; Hedges 2012), they undoubtedly belong to the amniote group, highlighting the fact that this organizational change likely suggests a reversion phenomenon (or secondary simplification).

To better understand the nucleolar organization in reptilian cells and especially the bicompartmentalized nucleoli which are poorly studied thus far, we will compare the bicompartmentalized nucleolus of turtle cells with that of lizard cells which has a tripartite organization. The study will be conducted mainly with primary liver and spleen cells from an aquatic turtle, Trachemys scripta scripta and a lizard cell line, Gekko gecko. We used different cytochemical (acetylation method, AgNOR staining) and immunocytological (TdT method for detecting DNA) techniques on the two reptile species and compared the results with those previously obtained in vivo (Lamaye et al., 2011). We also exposed the cells to different doses of drugs that affect rRNA and mRNA synthesis (Actinomycin D (AMD) and 5,6-dichlorobenimidazole 1- $\beta$-Dribofuranoside (DRB)) and studied their effects to investigate the link between structure and function in bicompartmentalized nucleoli. Finally, using BrUTP incorporation, we followed the volumic organization of rRNA during the time course of their synthesis and maturation within reptile nucleoli by means of laser scanning confocal microscopy coupled to three-dimensional image reconstructions, electron microscopy and double labelling with UBF. Our results clearly indicate that turtle nucleoli have different dynamics and react differently to drugs compared to classical nucleoli.

\section{Materials and methods}

\subsection{Cell cultures}

Turtle cell lines (Trachemys scripta scripta) were created in our laboratory and derived from liver and spleen fragments obtained after euthanasia. Homogenous cultures were obtained by repeated and forced mincing, frequently washed tissue fragments through a sterile screen and separating the resulting cells by centrifugation. Both cells were maintained in culture for 5 weeks in RPMI 1640-based media supplemented with foetal bovine serum. They attached to the T flash substrate as individual cells and aggregates and spread out 14 days after being placed in media. A lizard cell line (Gekko lung-1 [ATCC ${ }^{\circledR}$ CCL$\left.111^{\mathrm{TM}}\right]$ ) was purchased at ATCC Company. Turtle and Lizard cell lines were grown in $\mathrm{F} 12 \mathrm{~K}$ medium (Gibco) with $2 \mathrm{mM}$ L-glutamine adjusted to contain $1.5 \mathrm{~g} / \mathrm{L}$ sodium bicarbonate and $12 \%$ or $15 \%$ of foetal bovin serum respectively.

Actinomycin D (AMD) was added in the medium at a final concentration of $0.05 \mu \mathrm{g} / \mathrm{mL}$. All cell lines were incubated with the inhibitor for $30 \mathrm{~min}, 1 \mathrm{~h}$ and $2 \mathrm{~h}$.

5,6-dichlorobenimidazole 1- $\beta$-D-ribofuranoside (DRB) was dissolved in the medium at a final concentration of $100 \mu \mathrm{g} / \mathrm{mL}$. All cell lines were incubated with the inhibitor for $6 \mathrm{~h}$.

\subsection{Electron microscopy}

Small fragments of several tissues were fixed for $60 \mathrm{~min}$ at $4{ }^{\circ} \mathrm{C}$ in $1.6 \%$ glutaraldehyde in $0.1 \mathrm{M}$ Sorensen's buffer ( $\mathrm{pH} 7.4$ ), acetylated as previously described (Wassef et al., 1979) or stained with AgNOR technique (Ploton et al., 1984). After washing in Sorensen's buffer, cells were dehydrated through graded ethanol solutions and then processed for embedding in epon. Some fragments were fixed for $20-40 \mathrm{~min}$ at $4{ }^{\circ} \mathrm{C}$ in $4 \%$ formaldehyde in $0.1 \mathrm{M}$

Sorensen's buffer (pH 7.4) and embedded in Lowicryl K4M using the technique of Roth et al. (1981). Ultrathin sections were mounted on colloidin-coated grids, and stained with uranyl acetate and lead citrate before examination in a JEM 1400 transmission electron microscope at $80 \mathrm{kV}$.

\subsection{Detection of DNA: In situ terminal deoxynucleotidyl transferase (TdT)- immunogold method (Thiry, 1992)}

Acetylated ultrathin sections were incubated for $30 \mathrm{~min}$ at $37^{\circ} \mathrm{C}$ on the surface of the following medium: $100 \mathrm{mM}$ sodium cacodylate $(\mathrm{pH}$ 6.5), $10 \mathrm{mM}$ beta-mercaptoethanol, $2 \mathrm{M} \mathrm{MnCl}_{2}, 50 \mu \mathrm{g} / \mathrm{ml}$ bovine serum albumin (BSA) (sigma, USA), $20 \mu \mathrm{M}$ 5-bromo-2-deoxyuridine (BUdR) triphosphate (Sigma, USA), $4 \mu \mathrm{M}$ each of dCTP, dGTP, and dATP (Boehringer Mannhem, Germany) and $125 \mathrm{U} / \mathrm{ml}$ TdT (Boehringer; Mannheim, Germany). After three rinses in double-distilled water, the different sections were incubated for $20 \mathrm{~min}$ in PBS $(0.14 \mathrm{M} \mathrm{NaCl}, 6 \mathrm{mM}$ $\mathrm{Na}_{2} \mathrm{HPO}_{4}, 4 \mathrm{mM} \mathrm{KH} \mathrm{PO}_{4}, 1 \% \mathrm{BSA}, \mathrm{pH} 7.2$ ) containing normal goat serum (NGS) diluted 1:30. After rinses in PBS containing 0.2\% BSA, pH 7.2 , the next step of the treatment was a $4 \mathrm{~h}$ incubation at room temperature with mouse anti-BUdR antibodies (Roche, USA) diluted 1:50 in PBS pH 7.2 containing 0.2\% BSA and $0.2 \%$ NGS. After four rinses in PBS containing $1 \% \mathrm{BSA}, \mathrm{pH} 7.2$ and one in PBS containing $0.2 \% \mathrm{BSA}, \mathrm{pH}$ 8.2 , sections were transferred to an incubation medium containing rabbit anti-mouse IgG coupled to colloidal gold (10-nm diameter, Amersham Life Science, Belgium) diluted 1:40 in PBS (with 0.2\% BSA), $\mathrm{pH} 8.2$, and incubated for $1 \mathrm{~h}$ at room temperature. Samples were rinsed with $\mathrm{PBS}$ containing 1\% BSA, $\mathrm{pH} 8.2$ four times, then four times with distilled water.

Several kinds of control experiments were carried out. First, TdT or labelled nucleotides were omitted from the TdT medium and in second control, the primary antibody was omitted.

\subsection{Transfection and BrUTP incorporation}

Transfection reagents used for BrUTP incorporation in turtle and lizard cell line were Xtreme-GENE 9 and Xtreme-GENE HP respectively. We followed the protocol described in Thiry et al. (2008) and performed chases of $5,15,30,60$ or $120 \mathrm{~min}$.

\subsection{Immunolabelling}

After BrUTP incorporation, each well was fixed for $10 \mathrm{~min}$ at room temperature in $2 \%$ formaldehyde in buffered PBS ( $\mathrm{pH} 7.4$ ) and washed 
three times with PBS. A solution of Triton X-100 0.3\% in PBS was added to each well for $15 \mathrm{~min}$ followed by three washes of PBS. The preparation was blocked by normal goat serum (NGS) diluted 1:20 in PBS and then indirectly immunolabelled for $30 \mathrm{~min}$ at $37^{\circ} \mathrm{C}$ by mouse antiBUdR antibodies (Roche, USA) and rabbit antiserum against recombinant full length Xenopus UBF (Cairns and McStay, 1995) provided by Prof. Brian McStay (School of Natural Sciences Galway, Irland) both diluted 1:100 in PBS (pH 7.2). The staining was revealed during $30 \mathrm{~min}$ at $37^{\circ} \mathrm{C}$ by secondary goat anti-mouse and goat anti-rabbit antibody and marked with DAPI diluted 1:250 and 1:50 000 respectively in PBS.

The preparations were examined using an Olympus IX71. For the three-dimensional reconstruction, acquisitions were made using a Zeiss LSM 710 NLO and processed with the Amira software.

\subsection{Immunofluorescence methods}

The slides were fixed for $10 \mathrm{~min}$ at room temperature in $4 \%$ formaldehyde in $0.1 \mathrm{M}$ PBS ( $\mathrm{pH}$ 7.4). Then, they were permeabilized for 10 min in $100 \%$ methanol, rehydrated, rinsed in PBS, and further rinsed in PBS containing $1 \%$ BSA (w/v) and normal goat serum (NGS) diluted $1 / 30$. The cells were placed for $30 \mathrm{~min}$ at $37^{\circ} \mathrm{C}$ with a monoclonal antiBromodeoxyuridine (BrdU) antibody (Roche Diagnostics) diluted 1/100 in PBS, containing NGS diluted $1 / 50$ and $0.2 \%$ BSA. After rinsing with PBS containing $1 \% \mathrm{BSA}$, the slides were incubated for $30 \mathrm{~min}$ at $37^{\circ} \mathrm{C}$ with FITC-conjugated goat anti-mouse antibody (Sigma) diluted 1/100 in PBS containing $0.2 \%$ BSA.

After several rinses, the slides were mounted with Citifluor TM AF1 (Agar Scientific, Starsted, United Kingdom).

For the double labelling experiments, the slides were fixed for $5 \mathrm{~min}$ at room temperature in $4 \%$ formaldehyde in $0.1 \mathrm{M} \mathrm{PBS}(\mathrm{pH} 7.4)$ containing 1\% Triton X100. They were rinsed first in PBS, then in PBS containing $1 \%$ BSA, as well as NGS and normal sheep serum (NSS) both diluted $1 / 30$. They were incubated for $30 \mathrm{~min}$ at $37^{\circ} \mathrm{C}$ with an antiBrdU antibody and a rabbit polyclonal antibody against UBF (a gift from Prof Brian McStay, Galway, Ireland), both diluted 1/100 in $0.1 \mathrm{M}$ PBS containing $0.2 \%$ BSA, NGS, and NSS, both diluted $1 / 50$. The slides were washed with PBS containing 1\% BSA and incubated for $30 \mathrm{~min}$ at $37^{\circ} \mathrm{C}$ with an FITC-conjugated goat anti-mouse antibody (Sigma, for BrUTP) and a biotinylated sheep anti-rabbit antibody (Roche Diagnostics, for UBF) diluted $1 / 100$ in PBS containing $0.2 \%$ BSA. This secondary antibody was detected with streptavidin labelled with Texas Red (Amersham Life Science,

Little Chalfont, United Kingdom) diluted 1/100 in PBS containing $0.2 \%$ BSA for $10 \mathrm{~min}$. After rinsing, the cells were mounted with Citifluor TM AF1.

\subsection{Confocal microscopy}

A Biorad 1024ES system (Bio-Rad, Hercules, California) mounted on an inverted IX70 Olympus optical microscope was used. All acquisitions were made using a planapochromat 360, 1.4 numerical aperture oil immersion objective. Acquisitions were performed by exciting the FITC with the 488-nm line of a krypton/argon laser. The emission light was collected through a bandpass filter at $522-616 \mathrm{~nm}$. Texas Red was excited with the 568-nm line of the same laser. The emission light passed through a high-pass filter at $685 \mathrm{~nm}$ before collecting. Phase contrast images were collected simultaneously on a specific detector. For threedimensional investigations, 30 to 50 optical sections were recorded with a $0.25-\mu \mathrm{m}$ z-step from the top of the cell. Colocalization of the green and red signals was done in two ways. First, green and red images corresponding to a single optical section were classically overlaid and superimposition of both signals appeared in yellow. Second, a more accurate picture of the colocalization pattern was also obtained by using the Biorad 1024ES software. In this procedure, the intensity in the green channel was plotted against that of the red one for each pixel of both images. The scatter-plot thus obtained was used to select the pixels that display the highest levels of both green and red (i-e, between gray levels 126 and 255). These pixels, which correspond to a significant labelling of both species (i-e, to a real colocalization area) were shown in yellow relative to all the other pixels (that indicated either a single labelling, or a low intensity of at least one of the labellings), which were represented in blue.

\subsection{Three-dimensional visualizations}

Files were transferred to a Sun Sparc20 workstation (Sun Microsystems, Mountain View, California) for processing, which was performed using the Analyze software (CNSoftware,

Southwater, United Kingdom) (Heliot et al., 1997; Klein et al., 1998). Volumes were obtained by resampling the number of sections to have an identical pixel size in the $\mathrm{x}, \mathrm{y}$, and $\mathrm{z}$ directions. A 33,333 cubic median filter was subsequently applied to remove background voxels that might have appeared in the final volumetric visualization pictures. From the reconstructed volumes, two visualization procedures could be used, both based on ray-tracing methods. On the one hand, the ray passed through the whole volume, and the value of the more intense voxel met through the ray was attributed to the resulting pixel. By scanning all the volume with the ray, a projection was obtained that allowed a volumic visualization. On the other hand, the ray was reflected when it met a voxel that had an intensity superior to a threshold. In contrast to the first method, it led to a surface visualization.

\subsection{Immunoelectron microscopy}

Ultrathin sections of epon prepared by the techniques of Thiry et al. (2008) were incubated with mouse anti-BrdU antibodies (Roche, USA) as described in Thiry et al. (2008).

\subsection{Quantitative evaluation}

The labelling density was calculated by program iTEM 5.2 and we used a minimum of 10 pictures randomly selected for each condition.

\section{Results}

\subsection{Nucleolar compartments in primary turtle cells}

To better characterize the nucleolar organization of turtle cells, we generated two primary cell cultures from the liver and spleen of an aquatic turtle, Trachemys scripta scripta. We also used as a comparative model the epithelial lung cell line from the lizard, Gekko gecko, which possesses a classical nucleolus with a tripartite organization.

To clearly distinguish the different nucleolar components, we first applied acetylation methods known to enhance the contrast of nucleolar structures and in particular to clearly visualize the intranucleolar clumps of condensed chromatin (Thiry et al., 1985). Under these experimental conditions, the turtle nucleoli are divided in two structural compartments (Fig. 1A and 1B). The fibrillar zone (FZ) is located centrally and made of fine fibrils. The FZ is surrounded by an irregular area made of granules, the granular zone (GZ). Inside the FZ, we can also observe a densely-contrasted material forming darker strands. The turtle nucleoli are also associated with condensed chromatin clumps. Most of the clumps are situated at the periphery but some small clumps can be detected inside the GZ, sometimes in close contact with the FZ. In contrast, in the lizard cells (Lamaye et al., 2011), the nucleoli are composed of three classical components. Several small FC are surrounded by a layer of electron dense fibrils known as the DFC. Both FC and DFC are included inside a much larger component of variable shape composed of granules: the GC. Perinucleolar and intranucleolar clumps of condensed chromatin can also be present.

To better understand the structural organization of the turtle 

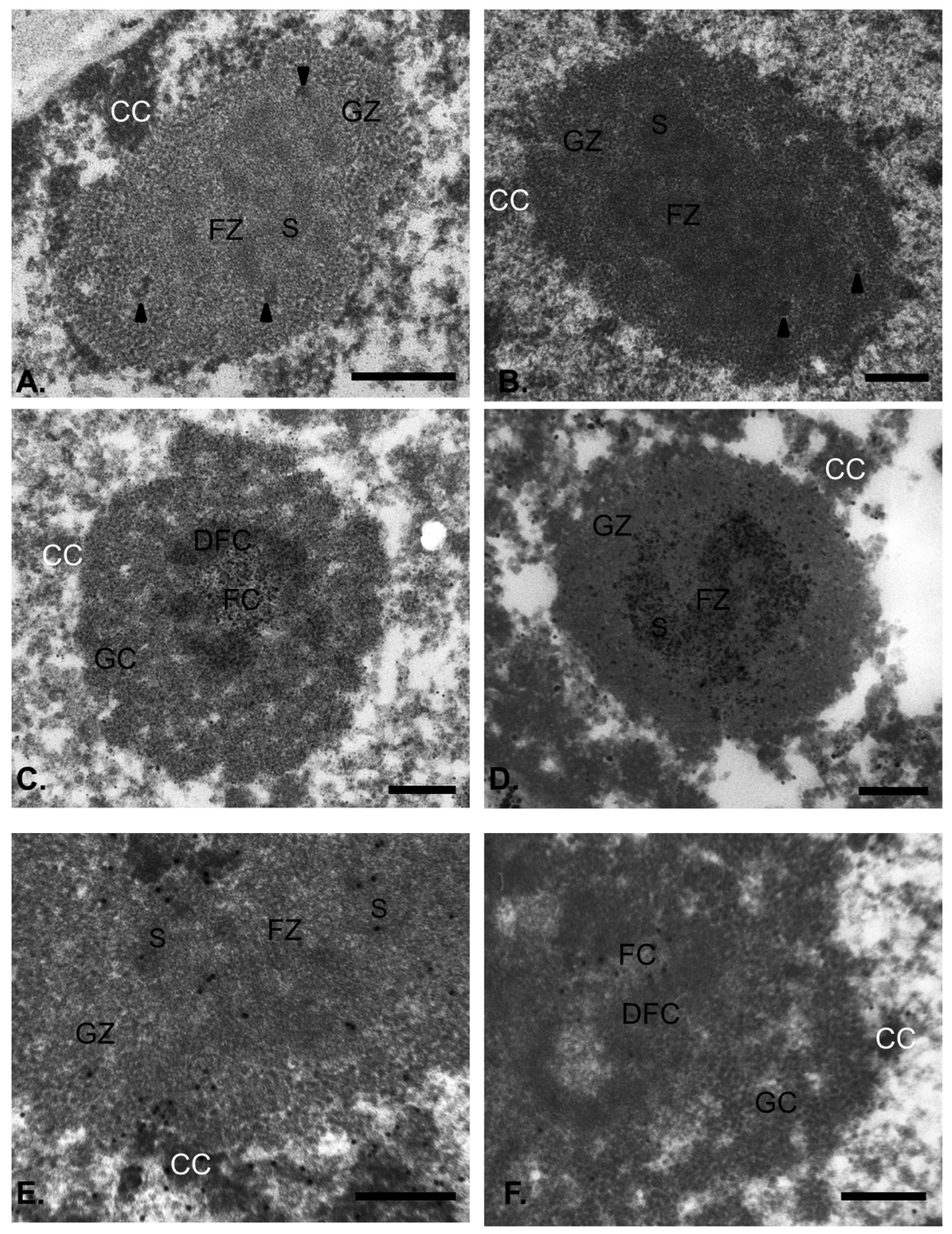

Fig. 1. Bipartite and tripartite organization of nucleoli in primary splenic (A, E) and liver (B, C) cells from a turtle Trachemys scripta scripta and in cell line from a lung epithelial cell from a lizard Gekko gecko (D, F) as revealed after the application of the acetylation method alone (A, B) or combined with the localization of AgNOR proteins (C, D) and DNA by the TdT technique (E, F). Arrowheads indicate intranucleolar clumps of condensed chromatin. CC: condensed chromatin, DFC: dense fibrillar component, FC: fibrillar center, GC: granular component, GZ: granular zone, FZ: fibrillar zone, S: dense strand. Bars $=1 \mu \mathrm{m}$.

nucleolus, we applied the AgNOR technique. In the nucleoli with tripartite organization, silver precipitates are preferentially deposited over the DFC and to a lesser extent over the FC. This is what we observed in the lizard nucleolus (Fig. 1C). In turtle nucleoli, the silver stain sets unevenly upon the FZ. It seems to set preferentially onto the dark strands (Fig. 1D).

To pinpoint the precise DNA location in turtle nucleoli, we used the TdT immunogold labelling procedure. In turtle nucleoli, an evident label is present over the intranucleolar and perinucleolar clumps of condensed chromatin. A labelling was also detected over the FZ, preferentially over the darker strands (Fig. 1E). A quantitative analysis of DNA labelling confirmed our qualitative observations (Fig. 1G). In particular, it showed that the labelling density over the darker strands was significant compared to that observed over the resin. In lizard cells, the gold particles were abundant over the condensed chromatin clumps. Inside the nucleolus, only FC's are significantly labelled while the DFC and the GC presented rarely gold particles (Fig. 2A-B).

\subsection{Effects of drugs on the nucleolar structure}

To clearly identify the various nucleolar components in turtle cells and to see the relationships between them, we used two drugs, AMD and DRB, well-known for reorganizing differentially the nucleolar components in tricompartmentalized nucleoli.

AMD at low doses is known to induce a segregation of nucleolar components. The three nucleolar components were organized always as follow: FCs, DFC and GC. The GC was devoid of any space and the condensed chromatin was in contact with FCs only. A typical nucleolar segregation was observed in the lizard cells treated for $2 \mathrm{~h}$ with AMD (Fig. 3A). In the turtle nucleoli of cells treated with AMD for 30-60 min (Fig. 3B-C), the FZ came in contact with the nucleolar surface. The electron dense cords gathered together, forming a dark mass in the central part of the FZ. The GZ did not surround completely the FZ. Intranucleolar clumps of condensed chromatin were visible at the transition site between the FZ and the GZ. In the turtle cells treated for 


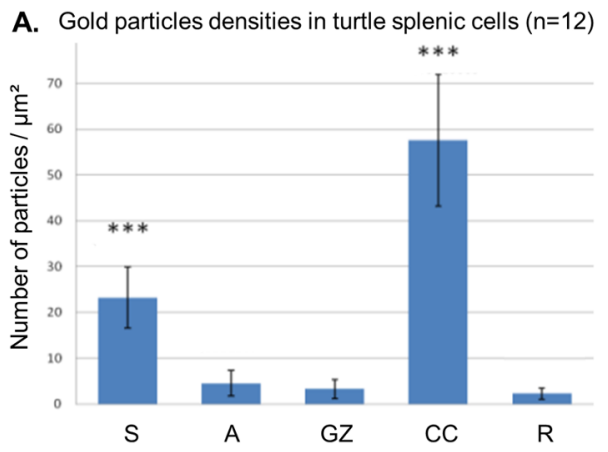

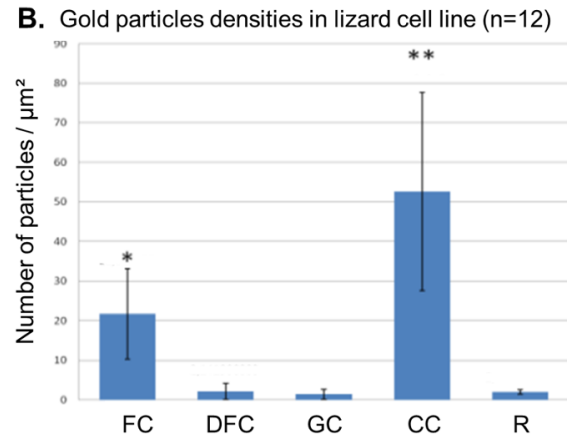

Fig. 2. Gold particles densities (number of particles per square micrometer) over the different nucleolar compartments in turtle splenic cells (A) and in lizard cell line (B). Student's $t$-test for nucleolar compartments vs. resin (*p<0.01, **p $<0.005$, $* * * \mathrm{p}<0.001)$. A: pale area, CC: condensed chromatin, DFC: dense fibrillar component, FC: fibrillar center, GC: granular component, GZ: granular zone, $\mathrm{R}$ : resin, S: dense strand.
$2 \mathrm{~h}$ with AMD (Fig. 3D), the condensed chromatin clumps disappeared from the nucleolus. Only several small clumps of condensed chromatin were maintained preferentially at the external surface of the FZ. The dark mass was always centrally located in the FZ. At this stage, the GZ kept its contact with the FZ.

DRB is known to cause a nucleolar fragmentation. The three compartments were dispersed inside the nucleoplasm and they did not keep any contact between them. This situation was seen in lizard cells treated for $6 \mathrm{~h}$ with DRB (Fig. 2E). In the two types of turtle cells, DRB did not provoke a nucleolar fragmentation but a redistribution of nucleolar components similar to that observed after AMD (Fig. 2F). The two main compartments segregated and the dark strands were condensed into a very dense mass in the center of the FZ. The intranucleolar clumps of condensed chromatin disappeared from the nucleolus and only the perinucleolar clumps persisted in close contact with the pale area of the FZ.

To better understand the nucleolar reorganization induced by drugs, we applied the TdT technique on AMD-treated cells. In both species of reptiles, the gold labelling is mainly found on condensed chromatin blocks (Fig. 2D). Regarding the lizard cell nucleolus, gold particles are found on FCs but rarely in the DFC. For the turtle cell nucleolus, several gold particles were detected on the dark mass inside the FZ and rarely in clear area of the FZ and in the GZ. Quantitative analysis of gold particles confirmed our qualitative observations (Fig. 4A-B). We also applied AgNOR staining on AMD-treated cells. In the segregated turtle nucleolus (Fig. S2A), the silver precipitates are concentrated mainly on
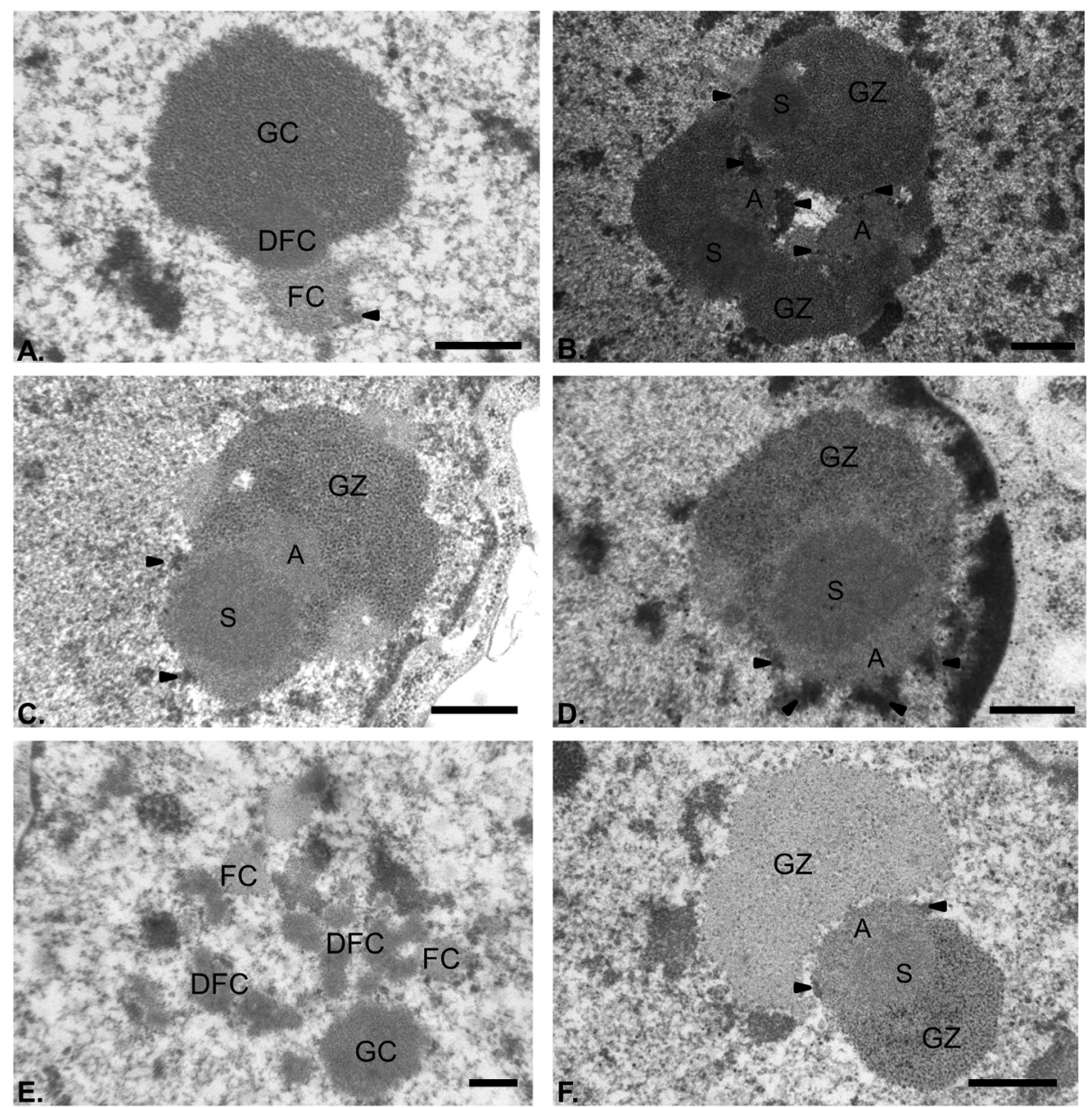

Fig. 3. Bipartite and tripartite organization of nucleoli in primary liver (A, C) and splenic (B, E) cells from a turtle Trachemys scripta scripta and in cell line from a lung epithelial cell from a lizard Gekko gecko (D, F) after a treatment with AMD for $30 \mathrm{~min}$ (A, C) and $120 \mathrm{~min}$ (B, D) or with DRB for $6 \mathrm{~h}$ (E, F). DFC: dense fibrillar component, FC: fibrillar center, GC: granular component, A: pale area, FZ: fibrillar zone, GZ: granular zone, S: dense strand. Arrowheads indicate small clumps of condensed chromatin in contact with the FC in the lizard nucleolus or with A of the FZ in the turtle nucleolus. Bars $=2 \mu \mathrm{m}$. 
A. Gold particles densities in turtle splenic cells treated with AMD $(n=12)$

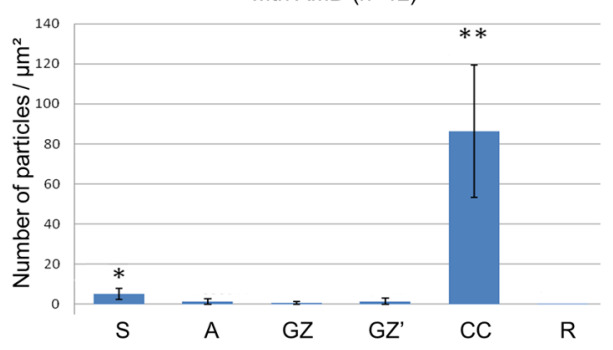

B. Gold particles densities in lizard cell line treated with AMD $(n=9)$

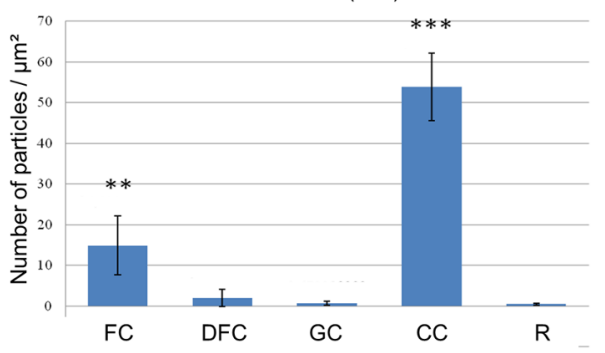

Fig. 4. Gold particles densities (number of particles per square micrometer) over the different nucleolar compartments in turtle splenic cells treated with AMD for $120 \mathrm{~min}$ (A) and in lizard cell line treated with AMD for $60 \mathrm{~min}$ (B). Student's $t$-test for nucleolar compartments vs. resin $(* \mathrm{p}<0.01$, $* * \mathrm{p}<0.005, * * * \mathrm{p}<0.001)$. A: pale area, CC: condensed chromatin, GZ: granular zone, R: resin, S: dense strand DFC: dense fibrillar component, FC: fibrillar center, GC: granular component. the central dense mass. In the segregated nucleolus of the lizard (Fig. $\mathrm{S} 2 \mathrm{~B}$ ), the silver staining is very intense on the CFD and lower on the CF.

\subsection{Dynamics of nascent rRNA inside the nucleolus}

To deepen the comparison between these two types of nucleolar organization, we conducted a pulse-chase experiment on our reptilian cell cultures to follow the dynamics of nascent rRNA inside the nucleolus using the technique previously described by Thiry et al. (2008) based on the incorporation of 5-Bromouridine-5'-triphosphate (BrUTP).

After $5 \mathrm{~min}$ of chase in the turtle nucleolus of splenic cells, we observed one or two groups of intense fluorescent foci (Fig. 5A). Each of them is formed by successive spots, giving the impression of a pearl necklace. The nucleoplasm was covered by a homogenous fluorescence
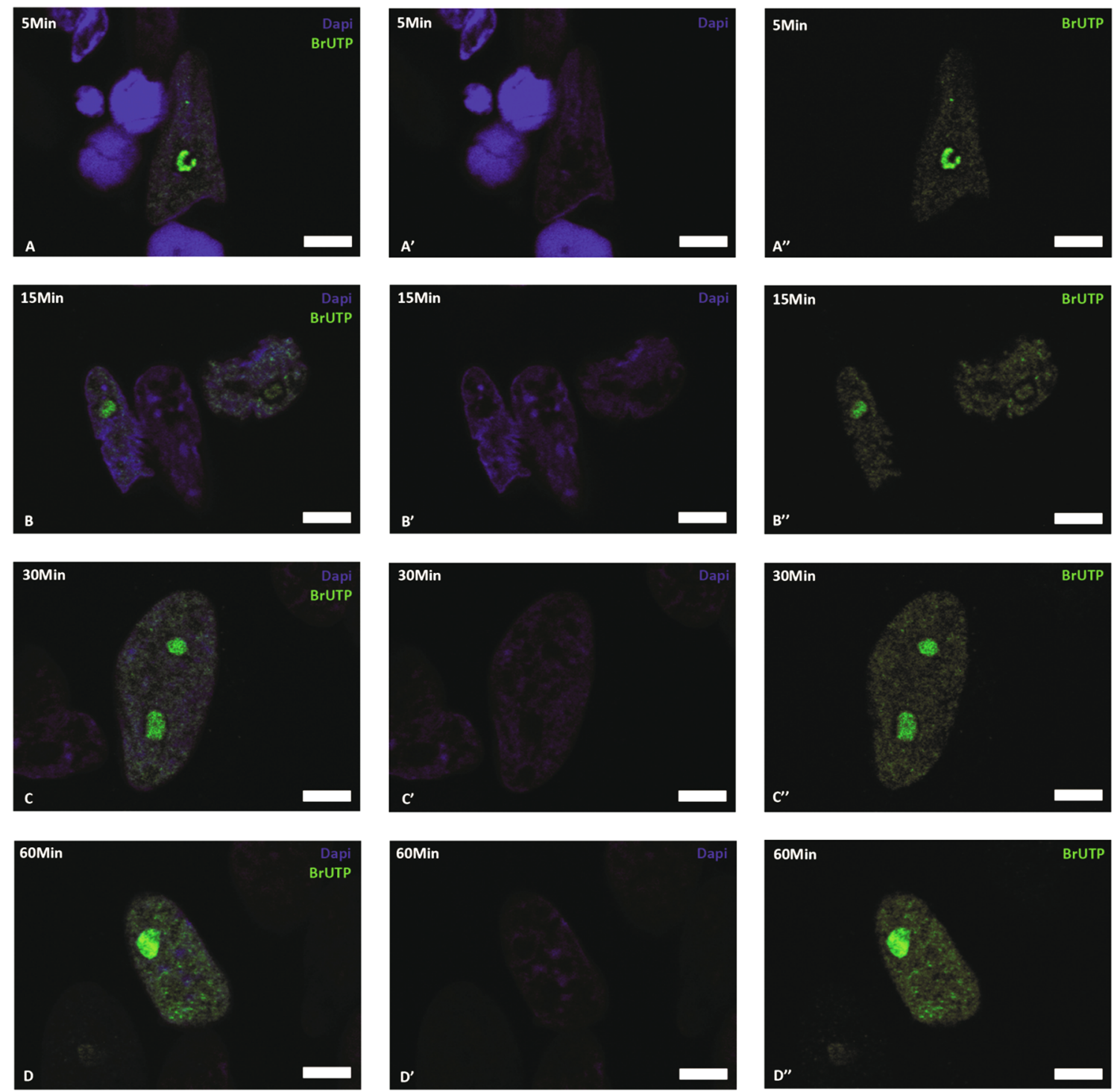

Fig. 5. Immunofluorescence localization of BrUTP-labelled RNAs in primary splenic cells. Cells were transfected for 15 min with BrUTP-Xtreme-GENE 9 complexes and then cultured for $5 \min (\mathrm{A}-\mathrm{A}$ ), $15 \mathrm{~min}$ (B-B"), $30 \mathrm{~min}$ (C-C") and $60 \mathrm{~min}$ (D-D”) before being fixed. BrUTP-labelled RNAs (green; A", B", C", D") were detected by an indirect immunofluorescence method. Dapi staining (blue) of the same fields obtained in confocal microscopy (A', B', C', D'). Overlay of green and blue signals (A, B, C, D). Bars $=5 \mu \mathrm{m}$. 
that never touched the necklace. The DAPI coated the nuclei except in some areas where we found intense foci with the two dyes separated by a negative circle. After a $15-30 \mathrm{~min}$ chase (Fig. 5B-C), pearls were transformed into spheres that covered almost all the negative space delineated by DAPI. The intensity of the nucleolar fluorescence was close to that of the nucleoplasm. After 60 (Fig. 5D) or $120 \mathrm{~min}$, the nucleolar label filled all the space devoid of DAPI. A weak label can be observed around the nuclei inside the cytoplasm. Similar observations were obtained in the turtle nucleolus of liver cells (Fig. S3).

The intranucleolar dynamics of ARN in the lizard cell nucleolus appear different from that obtained in the turtle nucleolus. It was very similar to that observed previously in the HeLa cell nucleolus (Thiry et al., 2000). After $5 \mathrm{~min}$ of chase (Fig. S4 A), lizard nucleoli showed two or three intense fluorescent spots generally found in its central part. Several small dots were spread across the nucleoplasm but rarely as intense as those found inside the nucleolus. The DAPI covered the nucleus except in areas where we found the intense fluorescent spots, where these two dyes never touch each other. After a $15 \mathrm{~min}$ chase, the nucleolar labelling formed a circle with a central circle devoid of labelling. This was particularly visible after a volumetric reconstruction of labels (Fig. S4 B). The nucleoplasm is covered by more fluorescent dots, smaller than before. After 30 min (Fig. S4 C), the nucleolar label became larger, sometimes approaching the DAPI signal, and less intense in the centre. The small dots covering the nucleoplasm were as intense as the mass found inside the nucleolus. After 60 or $120 \mathrm{~min}$, the nucleolar label filled completely the negative space delineated by DAPI, while still appearing empty in the centre.

\subsection{Time-dependent expansion of BrUTP-rRNA from UBF-positive sites to the periphery of the turtle nucleoli}

Having localized BrUTP incorporation, we attempted to determine whether this incorporation strictly takes place in sites where the rRNA machinery is located and whether the labelled RNA is rapidly excluded from these sites. To perform this study, the spatial distribution of prerRNA obtained in turtle splenic cells transfected with BrUTP for increasing times of chase was compared in individual optical sections to that of upstream binding transcription factor (UBF), a co-regulator of RNA polymerase I by constituting an initiation complex on rRNA genes (Cavanaugh et al., 2003; Goodfellow and Zomerdjik, 2012; Hamdane et al., 2014; Mangan et al., 2017).

After short chase periods (Fig. 6A-A"), BrUTP-RNAs (green) and UBF (red) display very close patterns in the nucleoli. Superimposition of these two images reveals a systematic colocalization of UBF with most transcripts (Fig. 6A). This result indicates that all the sites containing UBF are active for RNA synthesis. However, the transcripts occupy a much wider zone around UBF, suggesting a rapid depart of the transcripts from their transcription initiation sites. Fifteen minutes after transfection (Fig. 6B-B"), the relative surface of colocalization of the two signals strongly decreases and typically appears as mass centered
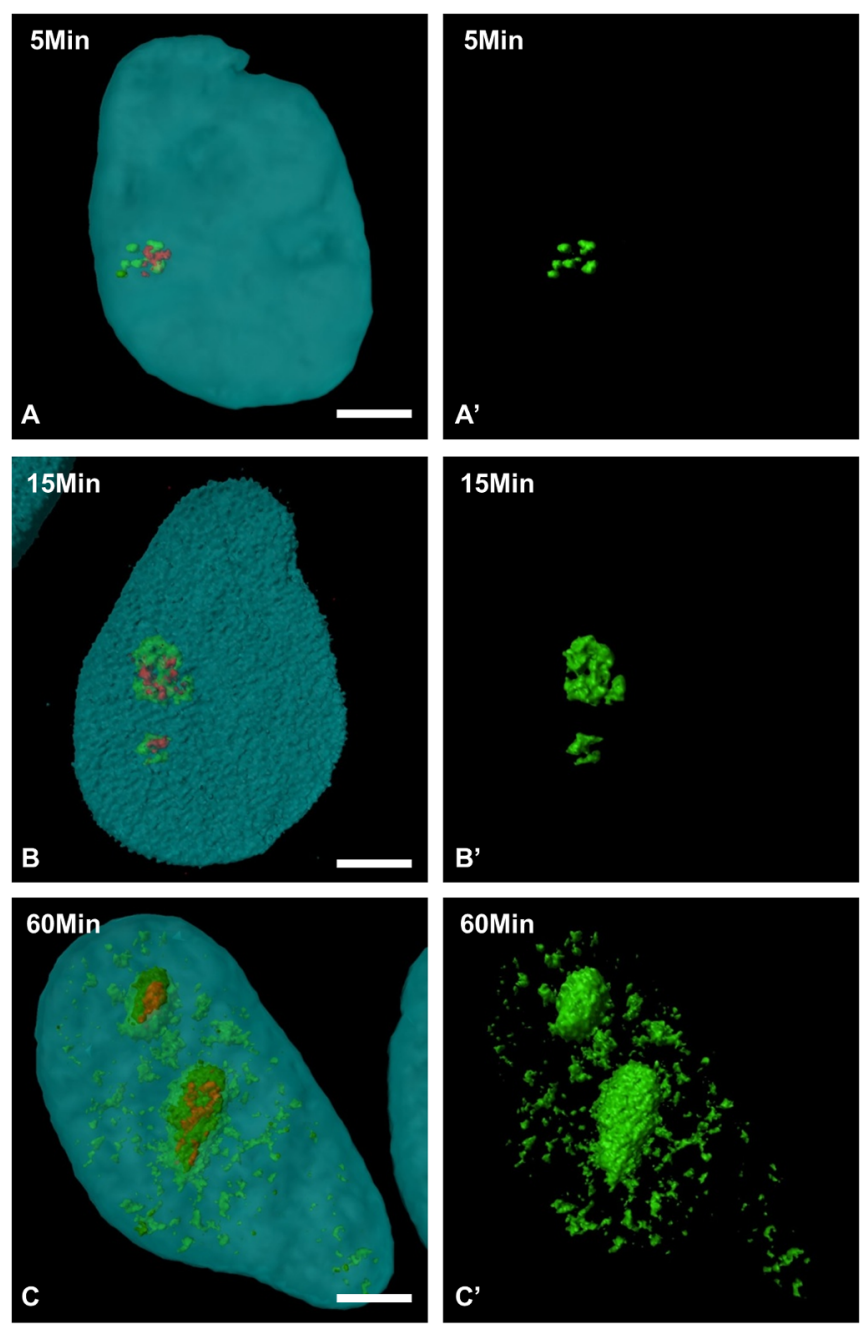
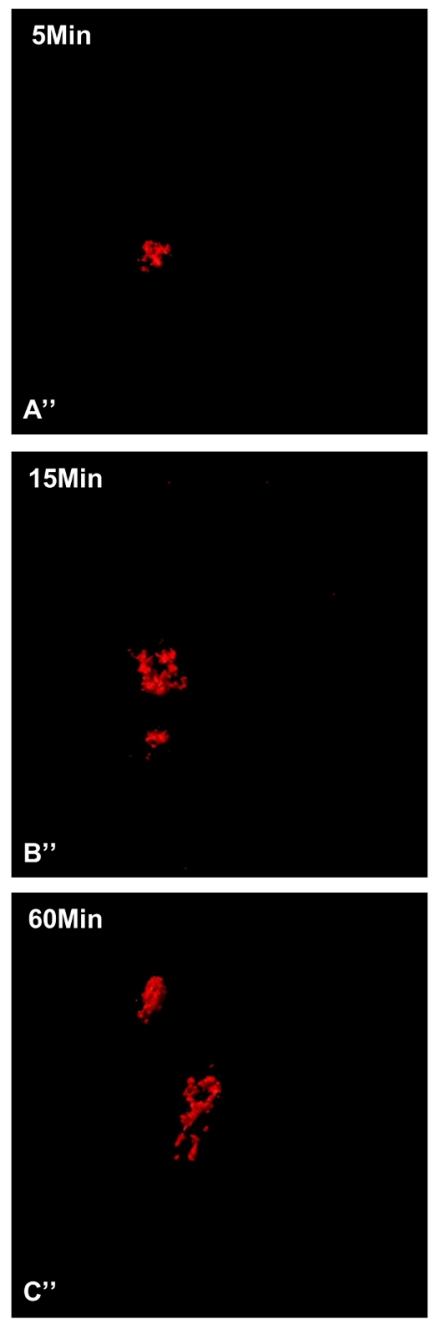

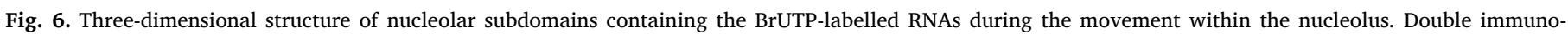

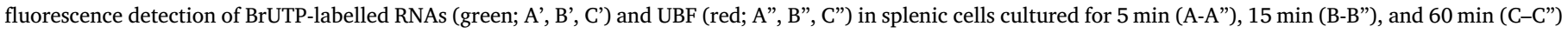
after transfection with BrUTP-Xtreme-GENE 9 complexes. Overlay of green and red signals (A, B, C). Bars $=5 \mu \mathrm{m}$. 

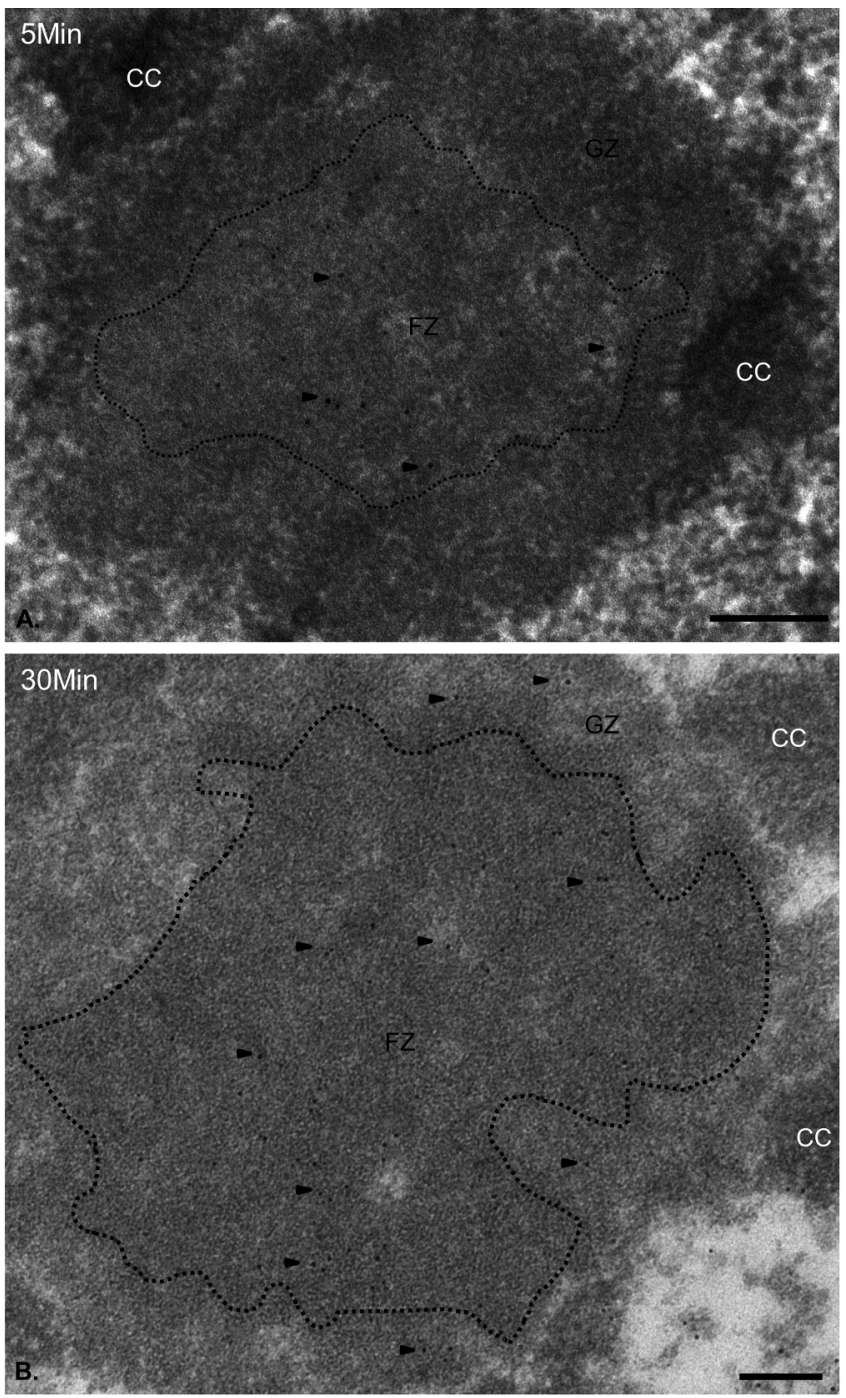

Fig. 7. Ultrastructural identification of nucleolar components containing the BrRNAs during their movement within the nucleolus. Immunogold labelling detection of BrUTP-labelled rRNAs on ultrathin sections of splenic cells transfected with BrUTP-Xtreme-GENE 9 complexes and then cultured in medium without BrUTP for $5 \mathrm{~min}$ (a) and $60 \mathrm{~min}$ (b). Under these experimental conditions, the two main nucleolar components are clearly identified: the granular zone (GZ) as well as the pale area and dark strands of the fibrillar zone (FZ, delimited by dotted line). CC: condensed chromatin. Arrowheads indicate gold particles. Bars $=0.25 \mu \mathrm{m}$.

on the sites at which UBF was located. BrUTP labelling seems to occupy an increasingly wider territory as the chase time increases. It is striking that BrUTP labelling does not disappear from the UBF-positive spots as the chase time increases (Fig. 6B). Finally, $1 \mathrm{~h}$ after transfection (Fig. 6C-C"), the colocalization areas are still present (Fig. 6C). It also appears that the number of UBF-positive sites in cells does not affect the dynamics of BrUTP-labelled RNAs from UBF-positive sites.

We are unable to reproduce the double immunolocalization experiment on lizard cells because the antibody anti-UBF used does not provide any labelling on this material.

\subsection{Ultrastructural identification of the nucleolar components containing BrUTP-RNAs during the extension within the turtle nucleolus}

To define the fine structural location of rRNA transcripts during their intranucleolar extension, our approach was applied at the ultrastructural level on the splenic cells from the aquatic turtle. Under these

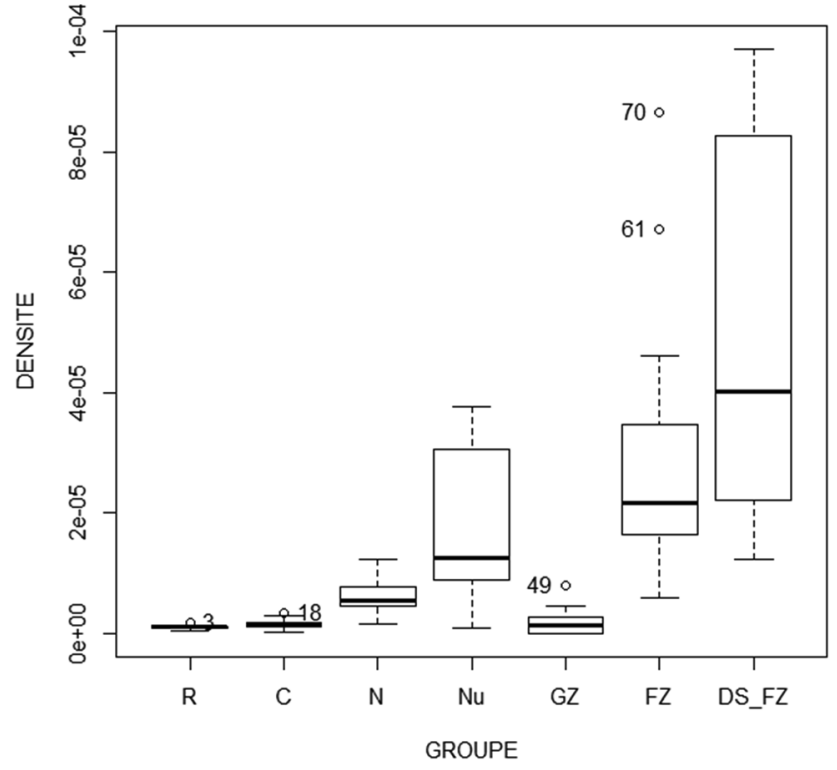

Fig. 8. Gold particles densities (number of particles per square micrometer) over the different cellular and nucleolar compartments in turtle splenic cells transfected with BrUTP-Xtreme-GENE 9 complexes and then cultured in medium without BrUTP for 5 min. C: cytoplasm, GZ: granular zone, FZ: fibrillar zone, N: nucleus, Nu: nucleolus, R: resin, DS: dense strand.

conditions, the ultrastructure of the nucleolus was well preserved; the two main nucleolar components were easy recognizable. After $5 \mathrm{~min}$ of chase (Fig. 7A), most of the gold particles were found in nucleoli while the nucleoplasm displayed only several labels. The nucleolar labelling occurred preferentially in the FZ. More precisely, gold particles were essentially present around and on dark strands. Quantitative analysis of gold particle densities in the different nucleolar components confirmed the presence of significant labelling over dark strands of the FZ (Fig. 8). After $30 \mathrm{~min}$ (Fig. 7B), the nucleolus was always the most intensely labelled site of cells. Nevertheless, the nucleoplasmic labelling was more evident. In the nucleolus, most gold particles were observed in the FZ, particularly over the dark strands (Fig. 7B). However, gold particles were also detected in the GZ.

\section{Discussion}

Our in vitro study on turtle cells confirms previous observations made on different tissues showing that the structural organization of the nucleolus differs from that of lizard and human cells (Lamaye et al., 2011; Thiry et al., 2000, 2011). The turtle nucleolus is essentially formed by two main compartments, a centrally-located FZ and a peripheral GZ, as observed in some invertebrates and anamniote vertebrates (Thiry and Lafontaine, 2005). It is also interesting to note that the bipartite organization of turtle nucleoli is not only a feature of aquatic turtle species but is also present in terrestrial turtles (Fig. S1 A).

A careful examination of the nucleolus reveals nevertheless that the FZ itself includes two parts. It contains cords formed by an electrondense material surrounded by an electron-lucid material. This structural organization does not appear to be characteristic of turtle cell nucleoli but typical of bicompartmentalized nucleoli. Indeed, the presence of electron dense cords within the FZ has already been noted in the nucleolus of different animal species (Thiry et al., 1991; Lamaye et al., 2011). It could be tempting to think that, with the dense strands of the $\mathrm{FZ}$, the turtle nucleolus is indeed also tricompartmentalized. But that is not true as there is no correspondence between zones that we observe in turtle and the one found in lizard or human as all our experiments highlight. Here, we show also that these cords are positive for the AgNOR staining and are the preferential site of DNA inside the 
nucleolus as revealed with the application of the TdT technique. We show also for the first time, that these cords represent the major sites of nucleolar labelling in turtle cells transfected with BrUTP and chased for short period. These results support the view that this part of the FZ could be the site of rDNA transcription in the turtle nucleolus.

Interestingly, in turtle cells, AMD induces a segregation of the two main compartments but it does not separate the two parts of FZ. The cords gather in the form of a very dense mass which is located in the center of the FZ. Moreover, we show that the DNA appears to leave these dense masses to find it in clumps of condensed chromatin on the periphery of the FZ. This observation is in agreement with previous observations made in the nucleolus of insect cells that showed the appearance of small clumps of condensed chromatin in the FZ following treatment with AMD (Thiry et al., 1991). It contrasts sharply with that observed in the segregated nucleoli of lizard or mammals in which the DNA persists in the fibrillar centers (Mikhaylova et al., 1996; Thiry et al., 1997).

Furthermore, treatment of turtle cells by DRB does not cause the separation of the functional compartments of the nucleolus as observed in mammalian and lizard cells (Zatsepina et al., 1996), but leads to a pattern identical to that obtained after AMD.

Finally, our study of the dynamics of nascent RNA within the turtle nucleolus also shows differences with that obtained in lizard and human cell nucleoli (Thiry et al., 2000). We observe an extension of labelling from the FZ towards the GZ, as already described in other bicompartmentalized nucleoli (Das et al., 1970). However, the labelling does not migrate from the UBF-positive sites, the dense cords in the FZ, to the maturation site. We see the maintenance of the labelling at the UBF-positive sites even after prolonged chases $(2 \mathrm{~h})$. This observation recalls highly localized tracks of specific transcripts within interphase nuclei visualized by in situ hybridization (Lawrence et al., 1989). These RNA tracks were also demonstrated be polarized structures that extended beyond the gene and showed a reproducible orientation with splicing domain (Xing et al., 1995). However, in a previous study made in HeLa cells, we have shown that the BrUTP labelling disappeared rapidly from the RNA polymerase I-positive sites as the chase time increased (Thiry et al., 2000). This result suggests that the kinetics of rRNA formation might be less ordered in the turtle nucleolus than in tricompartimentalized nucleoli.

Taken together, this data clearly indicates that the turtle nucleolus has a simpler structural and functional organization than that of the lizard and mammals, reminiscent of that of the bicompartmentalized nucleoli of anamniote vertebrates and invertebrates.

Although the exact origin of the different structural compartments of the nucleoli is not clear, it is tempting to speculate that these could come from two key steps in the formation of the two ribosomal subunits.

All transcription factors reside in the FC of the tricompartmentalized nucleoli (Thiry and Lafontaine, 2005). Pulse-chase experiments with BrUTP also showed that the primary transcript undergoing transcription rapidly penetrated into the DFC (Cheutin et al., 2002). The FC/DFC transition could thus represent the limit between transcription phenomena and the process of early maturation. This transition would not be visible in the bicompartmentalized nucleoli as there is no clear structure for each of these two phenomena; these two steps would not be separated in this type of nucleolus. On the other hand, in the two types of nucleoli, the DFC/GC or FZ/GZ transition could represent the place where the $90 \mathrm{~S}$ preribosomal particle splits into the two future preribosomal particles 60 and 40S. It is interesting to note that the same proteins are found in the GC of tricompartmentalized nucleoli and in the GZ of the bicompartmentalized nucleoli (Thiry and Lafontaine, 2005, Lamaye et al., 2011).

\section{Declaration of Competing Interest}

The authors declare that they have no known competing financial interests or personal relationships that could have appeared to influence the work reported in this paper.

\section{Acknowledgments}

The authors are grateful to B. McStay for the gift of an anti-UBF antibody.

\section{Funding}

This work is supported by the National Funds for scientific research (Belgium). DJ is a Postdoctoral research of the National funds for scientific research.

\section{Appendix A. Supplementary data}

Supplementary data to this article can be found online at https:// doi.org/10.1016/j.jsb.2019.09.015.

\section{References}

Boisvert, F.M., van Koningsbruggen, S., Navascués, J., Lamond, A.I., 2007. The multifunctional nucleolus. Nat. Rev. Mol. Cell Biol. 8 (7), 574-585.

Cairns, C., McStay, B., 1995. HMG box 4 is the principal determinant of species specificity in the RNA polymerase I transcription factor UBF. Nucleic Acids Res. 23 (22), 4583-4590.

Cavanaugh, A., Hirchler-Laszkiewicz, I., Rothblum, L., 2003. Ribosomal DNA transcription in mammals. In: Olson, M.O. (Ed.), The Nucleolus. Plenum Publisher, pp. 89-129.

Cheutin, T., O'Donohue, M.F., Beorchia, A., Vandelaer, M., Kaplan, H., Deféver, B., Ploton, D., Thiry, M., 2002. Three-dimensional organization of active rRNA genes within the nucleolus. J. Cell Sci. 115 (Pt 16), 3297-3307.

Chiari, Y., Cahais, V., Galtier, N., Delsuc, F., 2012. Phylogenomic analyses support the position of turtles as the sister group of birds and crocodiles (Archosauria). BMC Biol. 10, 65. https://doi.org/10.1186/1741-7007-10-65.

Das, N.K., Micou-Eastwood, J., Ramamurthy, G., Alfert, M., 1970. Sites of synthesis and processing of ribosomal RNA presurosrs within the nucleolus of Urechis caupo eggs. Proc. Natl. Acad. Sci. USA 67 (2), 968-975.

Derenzini, M., Pasquinelli, G., O'Donohue, M.F., Ploton, D., Thiry, M., 2006. Structural and functional organization of ribosomal genes within the mammalian cell nucleolus. J. Histochem. Cytochem. 54 (2), 131-145.

Goodfellow, S.J., Zomerdjik, J.C.B.M., 2012. Basic mechanisms in RNA polymerase I transcription of the ribosomal RNA genes. Subcell. Biochem. 61, 211-236.

Hamdane, N., Stefanovsky, V.Y., Tremblay, M.G., Németh, A., Paquet, E., Lessard, F., Sanij, E., Hannan, R., Moss, T., 2014. Conditional inactivation of upstream binding factor reveals its epigenetic functions and the existence of a somatic nucleolar precursor body. PLoS Genet. 10, e1004505.

Hedges, S.B., 2012. 2012Amniote phylogeny and the position of turtles. BMC Biol. 10 (64). https://doi.org/10.1186/1741-7007-10-64.

Heliot, L., Kaplan, H., Lucas, L., Klein, C., Beorchia, A., Doco-Fenzy, M., Menager, M. Thiry, M., O'Donohue, M.F., Ploton, D., 1997. Electron tomography of metaphase nucleolar organizers regions: evidence for a twisted-loop organization. Mol. Biol. Cell 8, 2199-2216.

Henras, A.K., Plisson-Chastang, C., O’Donohue, M.F., Chakraborty, A., Gleizes, P.E., 2015. An overview of pre-ribosomal RNA processing in eukaryotes. WIREs RNA 6, 225-242.

Hernandez-Verdun, D., 2006. The nucleolus: a model for the organization of nuclear functions. Histochem. Cell Biol. 126 (2), 135-148.

Klein, C., Cheutin, T., O'Donohue, M.F., Rothblum, L.I., Kaplan, H., Beorchia, A., Lucas, L., Heliot, L., Ploton, D., 1998. The three-dimensional study of chromosomes and of UBF-immunolabeled NOR's demonstrates their non-random spatial arrangement during mitosis. Mol. Biol. Cell 9, 3147-3159.

Koberna, K., Malinski, J., Pliss, A., Masata, M., Vecerova, M., Fialova, M., Bednar, J., Raska, I., 2002. Ribosomal genes in focus: new transcripts label the dense fibrillar components and form clusters indicative of "Christmas trees" in situ. J. Cell Biol. 157, 743-748.

Krüger, T., Zentgraf, H., Scheer, U., 2007. Intranucleolar sites of ribosome biogenesis defined by the localization of early binding ribosomal proteins. J. Cell Biol. 177, 573-578.

Lafontaine, D.L.J., 2004. Eukaryotic ribosome synthesis. In: Nierhaus, K. (Ed.), Protein synthesis and ribosome structure. Wiley-InterScience, pp. 107-143.

Lamaye, F., Galliot, S., Alibardi, L., Lafontaine, D.L., Thiry, M., 2011. Nucleolar structure across evolution: the transition between bi- and tri-compartmentalized nucleoli lies within the class Reptilia. J. Struct. Biol. 174 (2), 352-359.

Lawrence, J.B., Singer, R.H., Marselle, L.M., 1989. Highly localized tracks of specific transcripts within interphase nuclei visualized by in situ hybridization. Cell 57 (3), 493-502.

Mangan, H., Gailin, M.O., Mcstay, B., 2017. Integrating the genomic architecture of human nucleolar organizer regions with the biophysical properties of nucleoli. FEBS J. 284. https://doi.org/10.1111/febs.14108. 
Mikhaylova, V.T., Thiry, M., Stephanova, E., Goessens, G., Markov, D.V., 1996. Localization of nucleic acids in hepatocyte nucleoli of rats upon D-galactosamineinduced block of transcription. Exp. Cell Res. 225 (2), 389-398.

Müller, J., 2003. Early loss and multiple return of the lower temporal arcade in diapsid reptiles. Naturwissenschaften 90 (10), 473-476.

Ogawa, L.M., Baserga, S.J., 2017. Crosstalk between the nucleolus and the DNA damage response. Mol. BioSyst. 13 (3), 443-455.

Penzo, M., Montanaro, L., Treré, D., Derenzini, M., 2019. The ribosome biogenesis-cancer connection. Cells 8 (1).

Ploton, D., Menager, M., Adnet, J.J., 1984. Simultaneous high-resolution localization of Ag-NOR proteins and nucleoproteins in interphasic and mitotic nuclei. Histochem. J. 16 (8), 897-906.

Seither, P., Iben, S., Thiry, M., Grummt, I., 2001. PAF67, a novel protein that is associated with the initiation-competent form of RNA polymerase I. Biol. Chem. 382 (8), 1163-1170.

Thiry, M., 1992. Highly sensitive immunodetection of DNA on sections with exogenous terminal deoxynucleotidyl transferase and non-isotopic nucleotide analogues. J. Histochem. Cytochem. 40 (3), 411-419.

Thiry, M., Cheutin, T., O'Donohue, M.F., Kaplan, H., Ploton, D., 2000. Dynamics and three-dimensional localization of ribosomal RNA within the nucleolus. RNA 6 (12), $1750-1761$.

Thiry, M., Goessens, G., 1996. The nucleolus during the cell cycle. In: Landes, R.G. (Ed.), Molecular Biology Intelligence Unit. Springler-Verlag, Heidelberg, Germany, pp. $1-144$.

Thiry, M., Jamison, J.M., Gilloteaux, J., Summers, J.L., Goessens, G., 1997. Ultrastructural nucleolar alterations induced by an ametantrone/polyr(A-U) complex. Exp. Cell Res. 236 (1), 275-284.

Thiry, M., Lafontaine, D.L., 2005. Birth of a nucleolus: the evolution of nucleolar compartments. Trends Cell Biol. 15 (4), 194-199.

Thiry, M., Lamaye, F., Lafontaine, D.L., 2011. The nucleolus: when 2 became 3. Nucleus 289-293. https://doi.org/10.4161/nucl.2.4.16806.

Thiry, M., Lamaye, F., Thelen, N., Chatron-Colliet, A., Lalun, N., Bobichon, H., Ploton, D. 2008. A protocol for studying the kinetics of RNA within cultured cells: application to ribosomal RNA. Nat. Protoc. 3 (12), 1997-2004.

Thiry, M., Lepoint, A., Goessens, G., 1985. Re-evaluation of the site of transcription in Ehrlich tumour cell nucleoli. Biol. Cell 54 (1), 57-64.

Thiry, M., Schoonbroodt, S., Goessens, G., 1991. Cytochemical distinction of various nucleolar components in insect cells. Biol. Cell 72 (1-2), 133-140.

Turowski, T.W., Tollervey, D., 2015. Cotranscriptional events in eukaryotic ribosome synthesis. Wiley Interdiscip. Rev. RNA 6 (1), 129-139.

Wassef, M., Burglen, J., Bernhard, W., 1979. A new method for visualization of preribosomal granules in the nucleolus after acetylation. Biol. Cell 34, 153-158.

Werneburg, I., Sánchez-Villagra, M.R., 2009. Timing of organogenesis support basal position of turtles in the amniote tree of life. BMC Evol. Biol. 9, 82.

Xing, Y., Johnson, C.V., Moen Jr, P.T., McNeil, J.A., Lawrence, J., 1995. Nonrandom gene organization: structural arrangements of specific pre-mRNA transcription and splicing with SC-35 domains. J. Cell Biol. 131 (6 Pt. 2), 1635-1647.

Zatsepina, O.V., Schöfer, C., Weipoltshammer, K., Mosgoeller, W., Almeder, M., Stefanova, V.N., Jordan, E.G., Wachtler, F., 1996. The RNA polymerase I transcription factor UBF and rDNA are located at the same major sites in both interphase and mitotic pig embryonic kidney (PK) cells. Cytogenet. Cell Genet. 73 (4), 274-278. 